\title{
Effect of Rhythmic Music Auditory Stimulation On Tics Modulation in Tourette Syndrome: A Case Study
}

\author{
Sofia Scataglini ${ }^{1 *}$, Giuseppe Andreoni ${ }^{1}$, Marcello Fusca ${ }^{1}$ and Mauro Porta ${ }^{2}$ \\ ${ }^{1}$ Department of Design, Politecnico di Milano, Italy \\ ${ }^{2}$ Department of Neurology, Tourette Centre, IRCCS “Galeazzi”, Italy
}

Submission: August 11, 2017; Published: August 30, 2017

*Corresponding author: Sofia Scataglini, Department of Design, Politecnico di Milano, Via Durando 38/A, 20158 Milano, Italy, Tel: +39 (02) 2399-8881; Email: sofia.scataglini@polimi.it

\begin{abstract}
This study presents some preliminary and unexpected findings of the possible role of the music in Tourette Syndrome (TS) treatment in a single case study who first elicit this effect. The role of rhythmic music auditory stimulation on tics modulation was quantitively investigated using an integrated assessment that consist in collecting simultaneous and synchronized tic detection recordings by the wearable device on the patient's trunk and a video recording from a camera. The experimental conditions were tested in the hospital and a patient's home with and without the presence of an external examiner: in silence, environmental music, and earphones administered music. Clinical result demonstrated the effectiveness of $4 / 4$ rhythmic auditory stimulation in tics of high intensity. The administration of music through the earphones produces an isolation of the subject requiring more attention in himself with a consequent reduction of tic elicitation.
\end{abstract}

Keywords: Tourette syndrome; Wearable monitoring; Music cognition; Rhythmic auditory stimulation; Automatic tic detection

\section{Introduction}

Tourette Syndrome (TS) is a chronic neurologic disorder characterized by the childhood onset of multiple motor and phonic tics that wax and wanes over time [1]. The syndrome is common (1\%) and the aetiology is complex (mostly genetic) and $90 \%$ of people have co-morbid psychiatric disorders and reduced quality of life [2]. In facts Tourette syndrome is the most common cause of tics. Other tic disorders include transient tic disorder, chronic motor tic disorder and chronic vocal tics disorder. Transient tic disorder includes both motor and vocal tics with duration of less than a year, while the latter two include motor or vocal tics, but not both, for over a year. The age at onset of Tourette syndrome ranges from 2-21 years, with a mean of 5-7 years being common [2].

A distinctive issue concerning the assessment of TS is the difficulty in quantifying and classifying objectively its various clinical manifestations. Multiple variables such as frequency, number of tic-types, intensity, complexity, body distribution, suppressibility, and interference with normal activities are commonly considered to assess the severity of TS [3]. The evaluation of TS is currently carried out either through clinical examination or through patient reports based on self-assessment of tic disorder. The analysis of video-recordings is the standard reference for many TS studies [4]. Recently our research group proposed an automatic method based on wearable technology (WT) for monitoring and quantifying motor-tics caused by the TS [5] and a new protocol for an integrated (video+WT) assessment of TS patients [6].

During the experiments, an unexpected event occurred: while waiting for being sensorized and with an environmental music in the laboratory, in one patient, suddenly the motor tics showed certain synchronization with music rhythm. The role of musicotheraphy in TS therapy was already observed and discussed in literature $[7,8]$ but up to now no quantitative evidence of the phenomenon was found in the literature. Music is known to help mask to channel the energy of their tics [8] into a functional endeavour (Tourette energy), with ticcing disappearing when participating in music making. The most often cited example for this therapeutic effect occurs when drumming [9]. To investigate these effects of music in TS in different conditions, i.e. according to the other varying experimental conditions (clinical operator presence/absence during observations, music presence/absence and the modality of its administration) we defined a dedicated experimental protocol.

Here we present the preliminary and unexpected findings of the possible role of music in TS treatment in a case study 
of a single subject who first elicit this effect. The competent Institutional Ethical Committee approved the study. The subject was properly informed about testing procedures, personal data treating, aims of the research, and we provided the informed consent before participation.

\section{Case Report}

One subject, female, 28 years old, TS diagnosis was recruited for this preliminary study. Inclusion criteria were a former diagnosis of TS by a neurologist. The experimental activity was conducted both in the hospital environment (Department of Neurology, Tourette Centre, IRCCS “Galeazzi”, Milan, December 2011) in a controlled clinical condition, and at home in a more relaxed situation. The rationale of this choice is to elicit possible influence of stressing environmental conditions on the pathology outcomes.

We assessed the effect of rhythmic music auditory stimulation on tics modulation using the experimental protocol and a video questionnaire both in hospital and at home of the subject. Here we report a brief extract of the video questionnaire asked after all the experimental protocol (Examiner: E; Patient: P):

E: "Music auditory stimulation that induce tic stimulation is voluntary or involuntary?"

P: "Involuntary. I felt an internal stimulus that was not voluntary."

E: "Do you think that your tics were synchronized with the rhythm of the music?"

P: "Yes."

E: "Have you find any difference between the tests at home and in the hospital?"

P: "Without the examiner the tics were the same in both situations. Because the music was the same and my tics were synchronized with the auditory stimulation."

E: "Have you find any difference between the environmental music and music on through the earphones?"

P: "I noticed that I had less tics with the earphones."

E: "Do you like to sing during the acquisition?"

P: "Yes, I sang with earphones in order to try to decrease my tics and to be more concentrated. I felt isolated."

E: "If I changed the music, did you have the same tics?"

P: "No, it depends from the type of the music."

\section{Methods}

The experimental protocol consisted in a joint video and biosignal acquisition: the videotape protocol [10] involves four sessions of about 2.5 minutes. The patient was placed in a quiet room in front of a video camera (Figure 1). Two body views were recorded, full frontal body, and head and shoulders only under two conditions, relaxed with the presence of the examiner and relaxed with the patient alone. For this study, we used both the automatic system detection and the video-based procedure, referring to the full-frontal body observation in presence or without of the examiner.

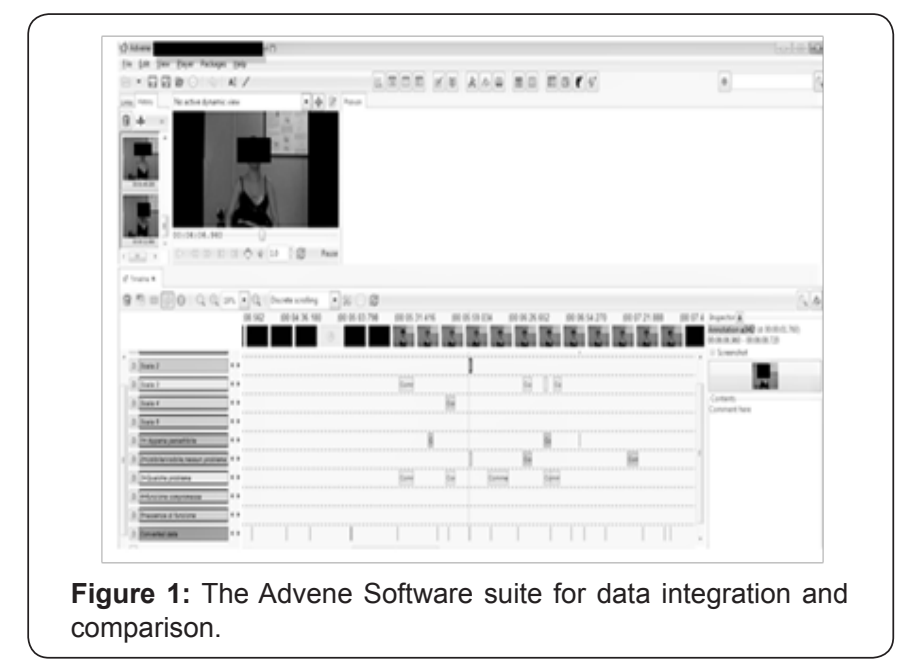

Two characteristics of the motor-tics were evaluated: intensity and frequency according to the 5-class scale proposed in literature [4]. According to the severity assessment proposed in the Rush Videotaping Rating Scale for the accelerometric assessment [5] we classified the motor TICS into a 5-levels scale:

1) Barely perceptible

2) Visible

3) Some problem

4) Impaired function

5) No function

A wearable device (WD) was placed through a belt on the chest of the subject for the automatic tic detection.

The commercial system (PROTHEO I, SXT - Sistemi per la Telemedicina, Lecco, Italy) consists of a plastic case containing a 1 ECG-lead and a 3D-acceleration sensor (LIS3L06AL, STMicroelectronics, Geneva, Switzerland), a Bluetooth transmission module, and a rechargeable Li-ion battery. The wearable device is supported by a software (HIM, Sensibilab, Politecnico di Milano, Lecco, Italy) designed for collecting and managing data in applications requiring real-time biosignal monitoring (Heart Rate) and movement (3D acceleration) [6].

Video analysis was carried out through an open source software (Advene, CNRS, France), providing a model and a format to share annotations about digital video documents (movies, courses, conferences...), as well as tools to edit and visualize the hypervideos generated from both the annotations and the audiovisual documents [11]. In this step of the research we focused on the correlations about experimental conditions and the patient behavior/ability to selectively respond to sound stimuli in terms of altered tic occurrence and their intensity following a structured multi factorial experimental protocol 
(Table 1). The music was chosen according to the patient Table1: Experimental Rationale. preferences (pop-rock music) with a $4 / 4$ time.

\begin{tabular}{|c|c|c|}
\hline \multicolumn{3}{|c|}{ Experimental Protocol } \\
\hline Research Question & Condition & Where \\
\hline \multirow{2}{*}{ Does environmental music affect tics occurrence? } & Standard test, Operator, Music & Hospital \\
\hline & Standard test, Operator, NO Music & Hospital \\
\hline \multirow{4}{*}{ Does examiner presence affect tics occurrence? } & Standing posture, Operator, NO Music & Hospital \\
\hline & Standing posture, NO Operator, NO Music & Hospital \\
\hline & Standing posture, Operator, NO Music & Home \\
\hline & Standing posture, NO Operator, NO Music & Home \\
\hline \multirow{4}{*}{ Does test place affect tics occurrence? } & Standard test, Operator, Music & Hospital \\
\hline & Standard test, Operator, Music & Home \\
\hline & Standard test, NO Operator, Music & Home \\
\hline & Standard test, NO Operator, Music & Hospital \\
\hline \multirow{3}{*}{ Does type of music administration affect tics occurrence? } & Standard test, Operator, NO Music & Home \\
\hline & Standard test, Operator, Environmental Music & Home \\
\hline & Standard test, Operator, Earphones Music & Home \\
\hline \multirow{4}{*}{$\begin{array}{l}\text { Is there a combined effect of clinical operator presence ad type } \\
\text { of music administration on tics occurrence? }\end{array}$} & Standard test, Operator, Environmental Music & Home \\
\hline & Standard test, no Operator, Environmental Music & Home \\
\hline & Standard test, Operator, Earphones Music & Home \\
\hline & Standard test, NO Operator, Earphones Music & Home \\
\hline
\end{tabular}

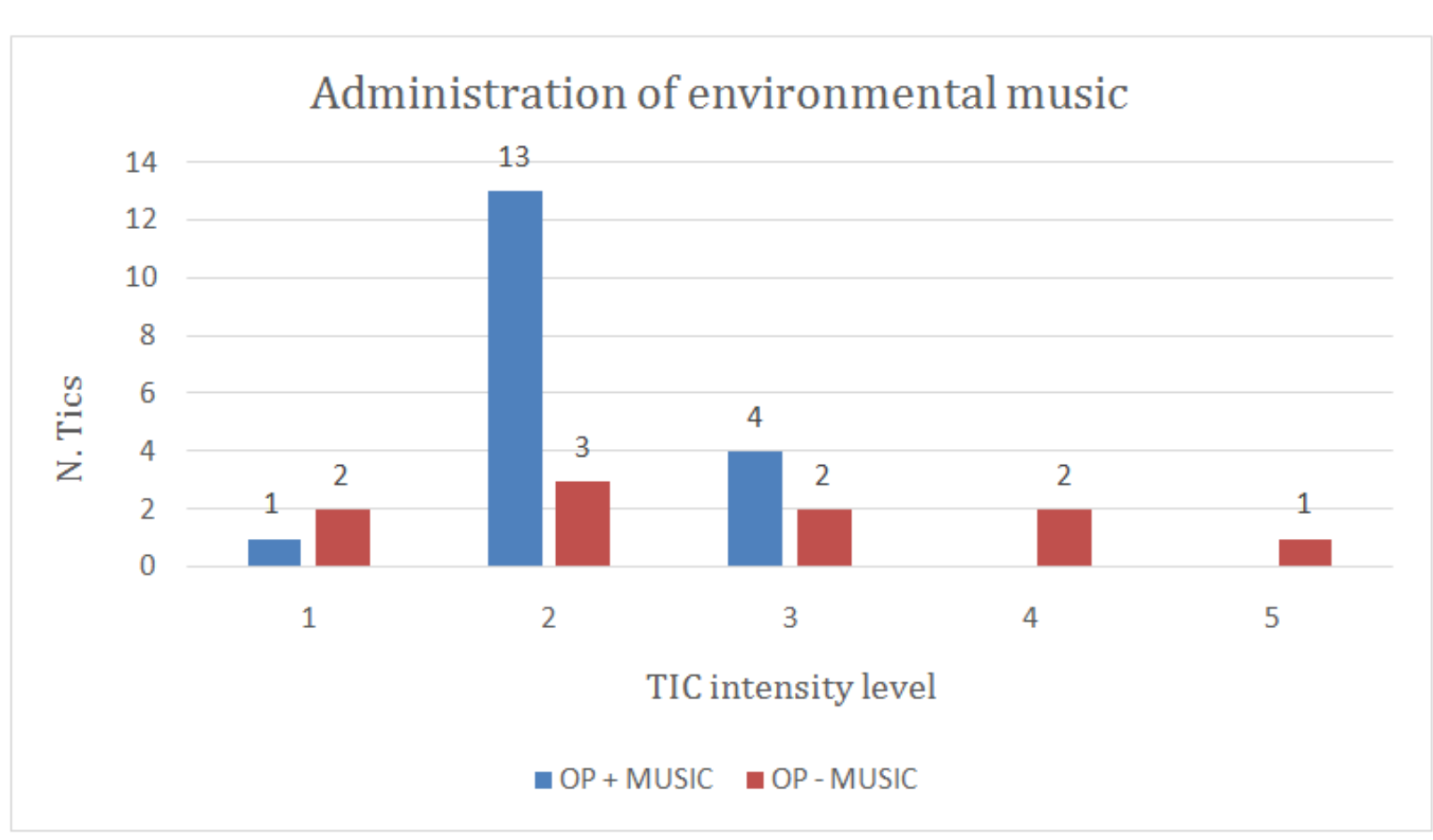

Figure 2: The role of environmental music on motor TIC elicitation.

\section{Results}

We analyzed the results in relation to the different research questions.

\section{Does environmental music affect tics occurrence?}

The recorded data seems to confirm that an environmental music can influence motor tics elicitation. With the presence of the clinical examiner in the laboratory, an $80 \%$ increase in the 
total number of tics in the subject. But this effect is related to low intensity tics probably because the patient tries to control the TS in presence of the direct observation of the operator for anxiety or to unconsciously obtain a better score, and to the effect of music synchronization (Figure 2). In fact, several tics occurred in correspondence of musical tone of the song, like a sort of "pulling" effect produced by music. More in detail, from video and accelerometric patterns analysis, the administration of environmental music, seemed to stimulate involuntary large amplitude body movements continuously synchronized with music like a sort of "dance".

\section{Does examiner presence affect tics occurrence?}

Data suggest that the presence of the examiner induces the patient to control her behavior thus increasing the number of low intensity tics; while the patient is alone in the laboratory, music induces a reduced but synchronized and high intensity motor tic as the subject seemed to dance (Figure 3). This factor must be combined also with the testing place. For this reason, we investigated the data related to the following research question no. 3.

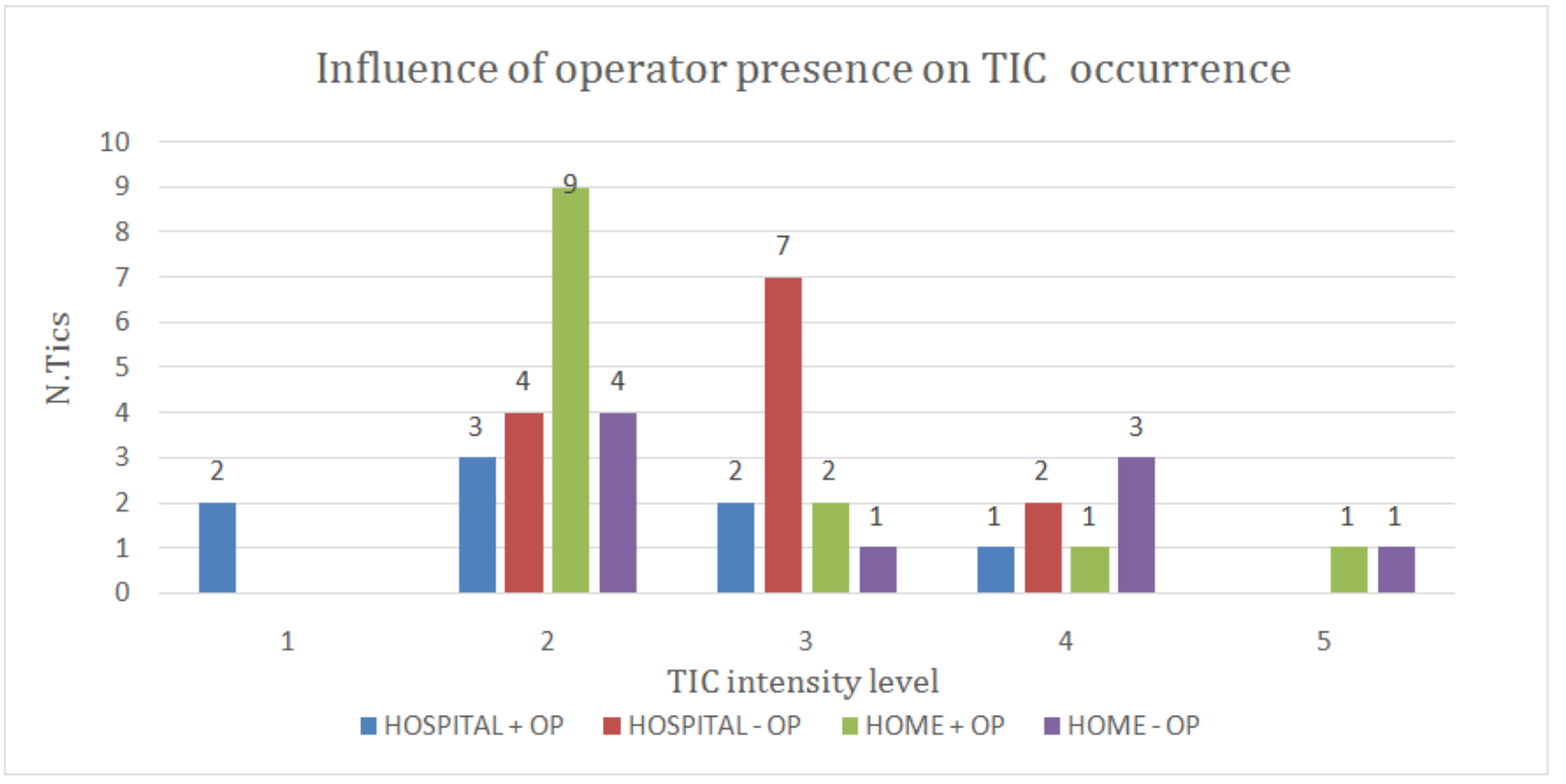

Figure 3: The role of the presence of the operator on motor TIC elicitation.

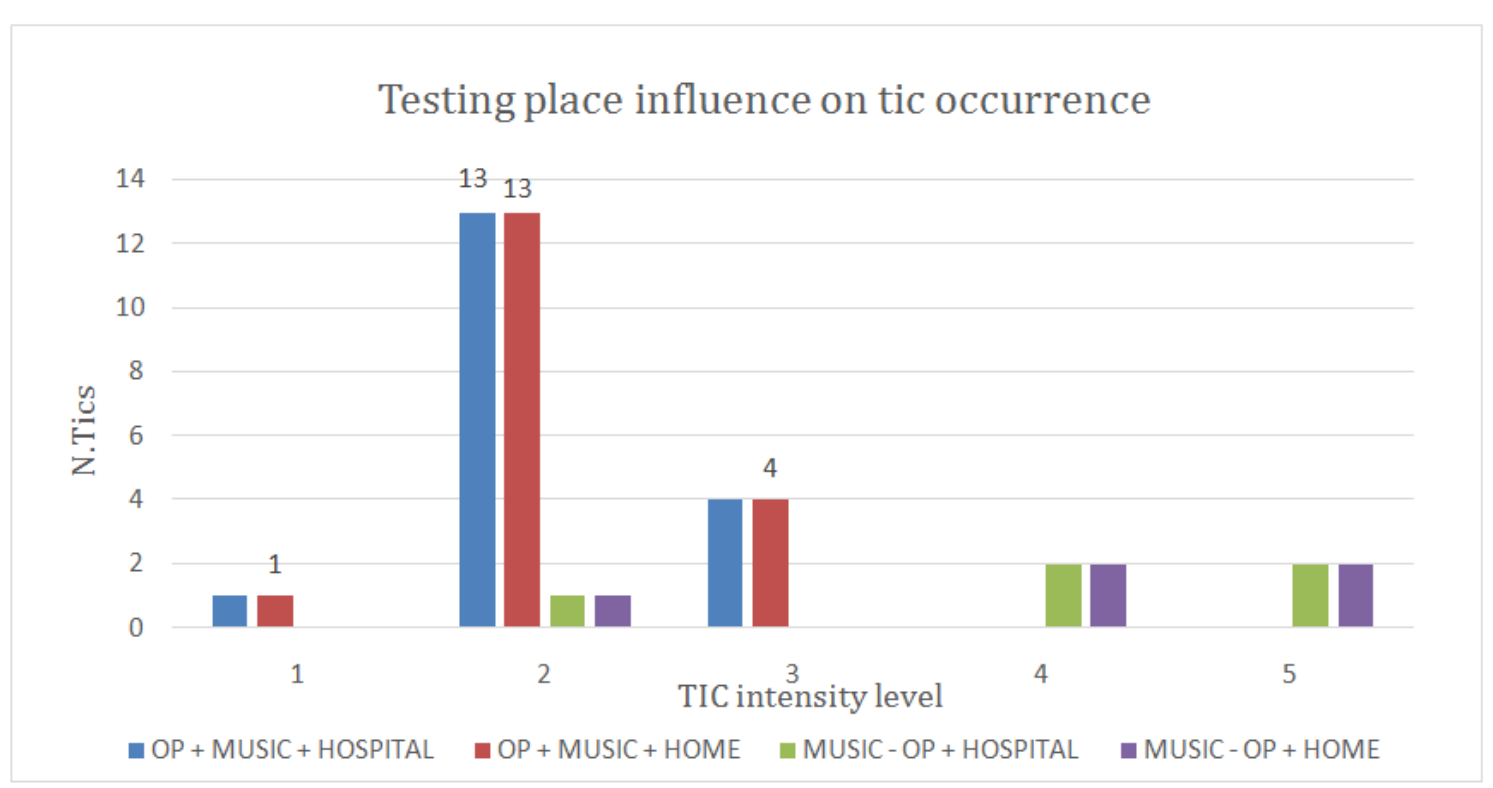

Figure 4: The role of testing place (Hospital laboratory vs Home room) on motor TIC elicitation. 


\section{Does test place affect tics occurrence?}

For better understanding this situation, we considered only the "place of test" factor, (Figure 4). No differences were found and music significantly reduced tic occurrence (-72\%) at home with respect to hospital. A controversial situation is shown concerning the presence of the clinical operator during the tests. In hospital, when the examiner is absent the resulting psychophysiological effort is reduced thus the tic number decrease but their intensity is greater. Data showed a 38\% reduction while the test is carried out in the hospital laboratory Figure 4 without examiner. The reverse happens at home: when the examiner observes the patient during the test a relevant increase of the number of tics $(+44 \%)$ is shown, those at lower intensity, probably for the attempt to control the events (psychological effort). Having demonstrated the effect of the presence of the operator during the exam and the role of the environmental music in the TS tic occurrence, we tried to administer music directly through a pair of earphones to investigate if this "direct stimulation" could more influence the phenomenon.

\section{Does type of Music administration affect tics occurrence?}

The administration of music through earphones seemed to be more effective. In a standard situation with the presence of the examiner, the environmental music stimulation proved to increase the total number of tics. Instead a direct stimulation through earphones allows a patient concentration on the music itself that significantly reduced the tic occurrence at each intensity level (globally -54\%), also those motor events at low intensity (Figure 5).

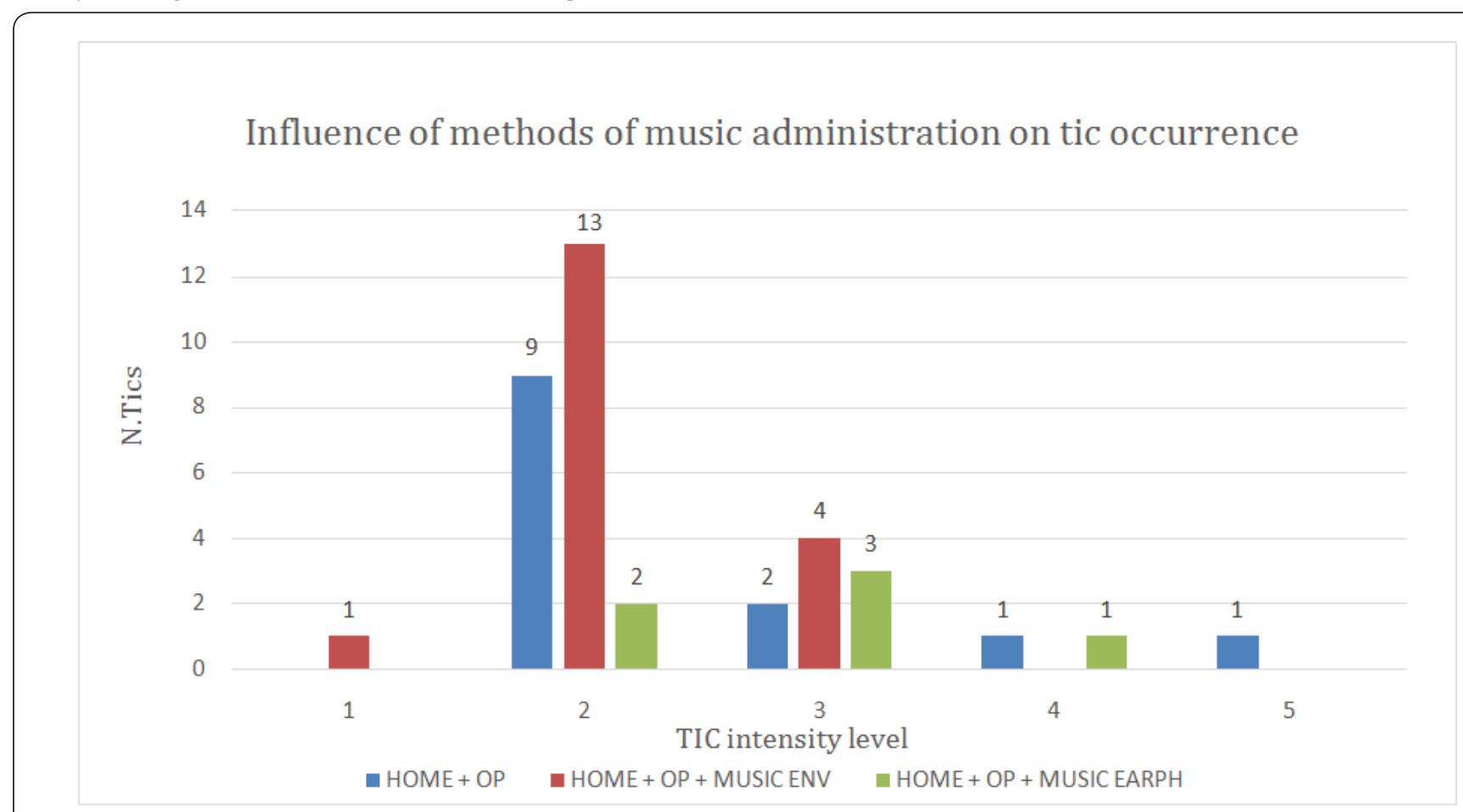

Figure 5: The earphone vs environmental music stimulation and their effect on motor tic elicitation.

Is there a combined effect of clinical operator presence ad type of music administration on tics' occurrence?

Finally, we tested a combined effect of environmental music and operator presence while carrying out the tests at home (Figure 6). The absence of the operator causes less stress with a diminution of tic numbers equal to $72 \%$. The earphones isolate the subject and then the number of tics is lowered significantly.
In tics of low intensity, the operator becomes not significant. The earphones isolate the subject and then the number of tics drops significantly. Both with the presence and in the absence of the operator we get the same results. The music on earphones reduces tic equal to $67 \%$, both with and without operator and this shows isolation of the subject. 


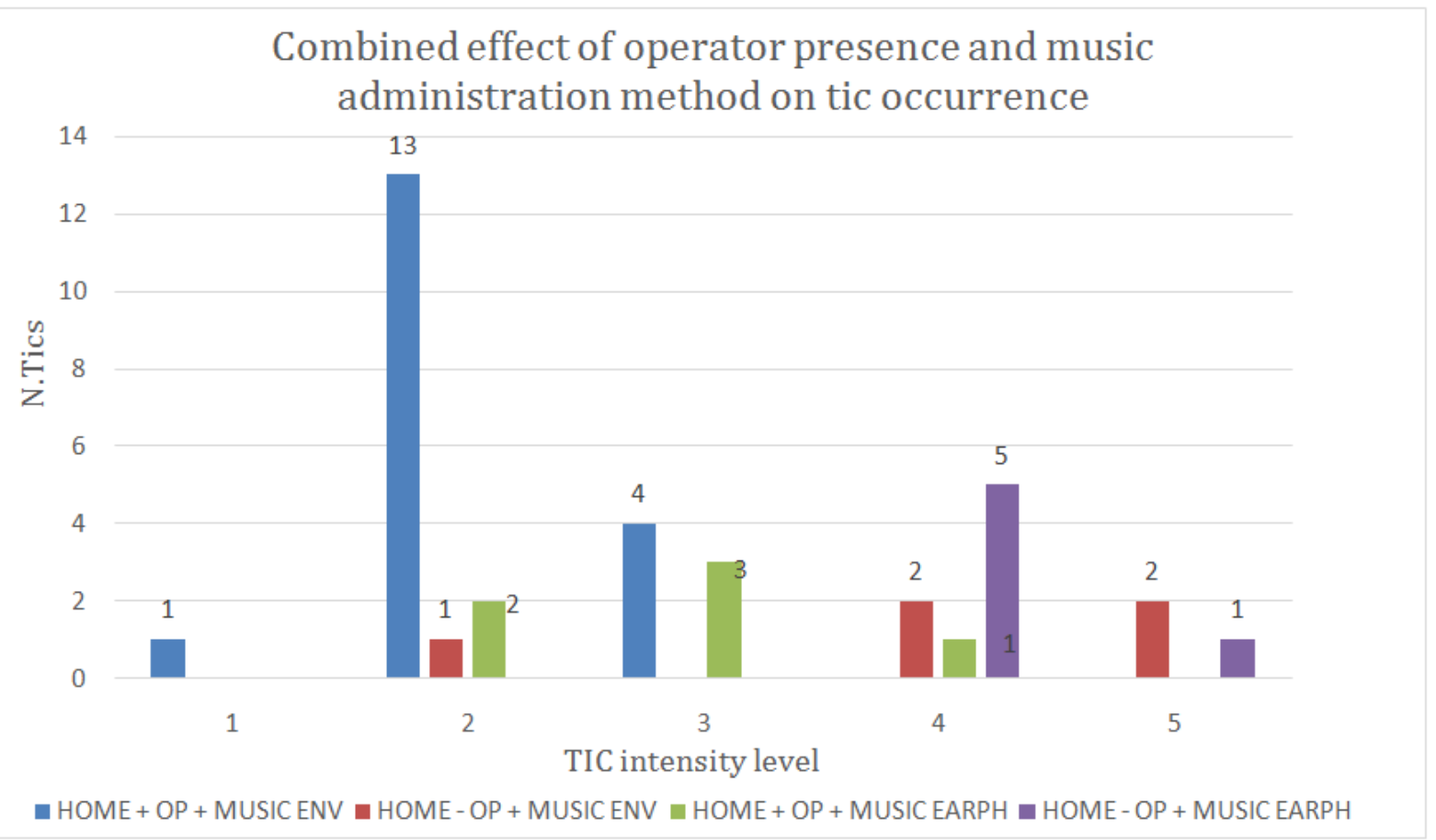

Figure 6: The addition of the effects of operator presence/absence and environmental music stimulation on motor TIC elicitation.

\section{Discussion}

This preliminary study aimed at verifying a possible influence of music in the elicitation of tics of TS. The tic is considerate as altered sensory input that gives as output a movement and behaviour (premonitory urges), [12]. This makes that from a sound stimulus may result in an altered motor behaviour. The 4/4 rhythmic music auditory stimulation with a strong beats accents reveals to be effective with a reduction of tics for high intensity that because the tic synchronized with rhythm as effect of neural entrainment $[9,13,14]$. The administration of the same rhythmic music auditory stimulation through the earphones produces an isolation of the subject requiring more attention in himself with a consequent reduction of tic elicitation.

\section{Conclusion}

Different factors were investigated to better understand also the modes of music administration to the subject and to establish a structured research protocol for further studies. The patient compliance was good even the protocol was long and complex. Now we will focus on actigraphy during long music administration and with different music rhythms and times.

\section{Referances}

1. Shapiro AK, Shapiro ES, Young JG, Feinberg TE (1988) Measurement in tic disorders". Gilles de la Tourette syndrome. Raven Press, New York, USA, pp. 452-480.

2. Robertson MM (2011) Gilles de la Tourette syndrome: the complexities of phenotype and treatment. Br J Hosp Med (Lond) 72(2): 100-107.

3. Himle MB, Capriotti MR, Hayes LP, Ramanujam K, Schell L, et al. (2014) Variables Associated With Tic Exacerbation in Children With Chronic Tic Disorders. Behav Modif 38(2): 163-183.
4. Goetz CG, Pappert EJ, Louis ED, Raman R, Leurgans S (1999) Advantages of a modified scoring method for the rush video-based tic rating scale. Mov Disord 14(3): 502-506.

5. Bernabei M, Preatoni E, Mendez M, Piccini L, Porta M, et al. (2010) A Novel Automatic Method for Monitoring Tourette Motor Tics Through a Wearable Device. Mov Disord 25(12): 1967-1972.

6. Scataglini S, Fusca M, Andreoni G, Porta M (2017) Application of Wearable Monitoring System in Tourette Syndrome Assessment. LNICST 192: 100-106.

7. Bodeck S, Lappe C, Evers S (2015) Tic-reducing effects of music in patients with Tourette's syndrome: Self-reported and objective analysis. J Neurol Sci 352(1-2): 41-47.

8. Sacks O (2007) Musicophilia: Tales of music and the brain. Random House, New York.

9. Large EW (2000) On synchronizing movements to music. Human Movement Science 19(4): 527-566.

10. Goetz CG, Tanner CM, Wilson RS, Shannon KM (1987) A rating scale for Gilles de la Tourette's syndrome: description, reliability, and validity data. Neurology 37(9): 1542-1544.

11. Aubert O, Prié Y (2005) Advene: active reading through hypervideo. Proceedings of ACM Hypertext 5: 235-244.

12. Leckman JF, Walker DE, Cohen DJ (1993) Premonitory urges in Tourette's syndrome. Am J Psychiatry 150(1): 98-102.

13. Henry MJ, Obleser J (2012) Frequency modulation entrains slow neural oscillations and optimizes human listening behavior". Proc Natl Acad Sci U S A 109(49): 20095-200100.

14. Nozaradan S, Peretz I, Moureaux A (2012) Selective neuronal entrainment to the beat and meter embedded in a musical rhythm. J Neurosci 32(49): 17572-17581. 
This work is licensed under Creative Commons Attribution 4.0 Licens

DOI: 10.19080/OAJNN.2017.5.555673
Your next submission with Juniper Publishers will reach you the below assets

- Quality Editorial service

- Swift Peer Review

- Reprints availability

- E-prints Service

- Manuscript Podcast for convenient understanding

- Global attainment for your research

- Manuscript accessibility in different formats ( Pdf, E-pub, Full Text, Audio)

- Unceasing customer service

Track the below URL for one-step submission https://juniperpublishers.com/online-submission.php 